\title{
A short-term vehicular density prediction scheme for enhanced beaconing control
}

\author{
Sofiane Zemouri, Soufiene Djahel and John Murphy \\ UCD School of Computer Science and Informatics, Ireland \\ sofiane.zemouri@ucdconnect.ie, \{soufiene.djahel,j.murphy\}@ucd.ie
}

\begin{abstract}
Channel congestion is a well-known problem in wireless networks in general and Vehicular Ad Hoc Networks (VANETs) in particular. Literature solutions propose to alleviate this problem by controlling the network load based on parameters like vehicle density or packet collision rate. In other words, each vehicle will observe the density of vehicles (or the packet collision rate) around itself in a certain time interval, and use this information to adjust its transmit parameters i.e. transmit rate and/or power, the next time it has a beacon to transmit (in the following time window). However, the information collected in the current time window might not still be valid in the next one. In fact, in a highly dynamic network like VANETs, vehicle density, and consequently the busy ratio and the collision rate, might vary a great deal even in the smallest time intervals. To cope with this newly identified problem, we propose a novel vehiclecentric short-term density prediction scheme that estimates the vehicular density around a given vehicle within the next time window allowing each vehicle to adapt its transmit parameters based on the current state of the network (as opposed to the previous state). The accuracy and the efficiency of our proposed scheme is demonstrated in a proof-of-concept case study, showing a significant improvement in terms of network performance.
\end{abstract}

Keywords-Adaptive Beaconing, Transmission Rate Control, Transmission Power Control, Density prediction, Density forecasting, VANETs, ITS.

\section{INTRODUCTION}

In Vehicular Ad Hoc Networks (VANETs), periodic beaconing is one of the essential blocks that enable all safety as well as non-safety applications. Beacons are short messages periodically broadcasted to all neighboring nodes acting as a pulse to the network. In addition to the information provided directly to different Intelligent Transportation Systems (ITS) applications, beacons play a key role in the synchronization of the network. In fact, vehicles use some information extracted from beacons to regulate their flows of messages and avoid congesting the network.

Packet collision can have catastrophic consequences on the performance of the network. In the absence of a proper packet loss recovery mechanism, VANETs cannot afford losing beacons. In addition, it is well known that packet collisions increase exponentially with the increase of vehicles' density. The limited communication medium, consisting in a set of reserved $10 \mathrm{MHz}$ radio channels located around the frequency band of $5.9 \mathrm{GHz}$, cannot support the heavy load inflected by vehicles in high-density scenarios like crowded cities. The high dynamicity, in terms of vehicle density, does not help fixing the network load problem in a VANET. This type of network is known to have no proper adaptation mechanism to regulate neither the number nor the extent of its messages. Many works have been proposed to cope with network congestion problem in VANETs, by adapting vehicle's transmission parameters (transmission rate and/or power), in order to avoid packet collisions and keep the channel at a good level of utilisation. ETSI ITS G5 has released a technical specification in this regard introducing Decentralised Congestion Control (DCC) [1], whose aim is to adapt beacons' Tx parameters to avoid channel congestion.

The main elements helping to adjust $\mathrm{Tx}$ parameters are the vehicle density observed around each vehicle, or the observed collision rate to a lesser extent. These elements are usually read out during fixed intervals called Synchronization Intervals (SI) during which all vehicles are expected to have accessed the channel at least once in order to broadcast their status messages (beacons). These readings are then used, as a base for each vehicle individually, to decide how often and how far should the beacons be broadcasted in the next SI. However, these readings might be outdated by the time they are used. In fact, it is known that VANETs are very dynamic networks in which, vehicles' densities may vary a lot even in the shortest time intervals. If we consider the granularity of $10 \mathrm{~Hz}$ (minimum beaconing interval required by the National Highway Traffic Safety Administration and the Crash Avoidance Metrics Partnership [2]), i.e. 10 beacons to be transmitted by each vehicle every second. At a crossroad, the density as seen by individual vehicles can vary drastically in as little as 100 milliseconds. This effect is further amplified by signal shadowing around corners, caused by buildings.

We argue that the information used to regulate the flow of beacons (density of vehicles and/or collision rate) in a VANET should reflect the current state of the network as opposed to the previously observed state in the past SI. Failing to respect this rule would result in an adaptation of Tx parameters on the basis of biased (outdated) data. We propose a short-term density prediction scheme that would estimate the state of the network during the next SI and therefore allows a fine-grained tuning of beacons' transmission parameters based on current network conditions.

In our previous work [3], we proposed a beacon adaptation scheme to cope with the channel congestion problem while allowing vehicles to have better awareness of their neighbourhood. This work builds up on the former to design a more solid congestion control mechanism and produce considerable enhancement in terms of network performance thanks to the novelty proposed in this work namely the short-term vehicular 
density prediction. The remainder of this paper is organized as follows: In section II, we will briefly present some of the related works. Section III will provide a detailed description of the proposed solution. The performance evaluation and the results are presented in section IV, and finally we conclude in section V.

\section{RELATED WORK}

As stated earlier in the previous section, many works have been proposed to cope with the network congestion problem in VANETs as the basic IEEE802.11p standard [4] does not provide any mechanisms to deal with this problem. Literature solutions can be grouped into three categories: (i) beacons transmit rate adaptation solutions, (ii) beacons transmit power adaptation solutions and (iii) hybrid solutions.

As an example for the first category, ATB [5] proposes to reduce the beaconing rate based on two key metrics: message utility and channel quality. Another example is DynB [6], which follows a similar approach but introduces the effects of shadowing caused by both buildings and cars on the wireless channel load. As an example for the second category (i.e. transmit power adaptation approach), [7] selects the transmit power according to the utility of the beacon to be transmitted. The authors in [8] follow a completely different criterion and propose to randomly select the transmit power of vehicles following a given probability distribution. Some researchers have proposed hybrid solutions like [9] - [11] where the transmit rate and power are adapted jointly.

In 2011, ETSI TS G5 introduced the DCC [1] (Decentralised Congestion Control). The idea behind this is to combine beacon rate and power adaptation with other forms of adaptations like the Carrier Sense Threshold. However, many works have followed like [12] and [13] pointing out the drawbacks of this solution. In our previous work [3], we introduced a new beacons control scheme by adapting the rate and the power in a smart way. We have achieved considerable enhancement in terms of network performance yet; we still see room for improvement.

In this new work, we introduce a short-term vehicle density prediction scheme to allow beaconing adaptation based on the real time situation of the network. To the best of our knowledge, this is the first work proposing such a prediction in this context for the purpose of beacons control.

\section{SHORT-TERM VEHICULAR DENSITY PREDICTION}

As stated earlier, our solution represents a short-term vehicle density prediction scheme for the purpose of regulating beacons transmission. In this section, we will explain the concept and present the prediction algorithm.

\section{A. Assumptions}

In this work, we focus on the broadcast of beacons in urban areas. It is known that the density of vehicles in such scenarios is relatively high. Since the wireless medium, on which beacons are broadcasted, has a limited capacity, it only allows a limited number of nodes (vehicles) to access the channel in every CCHI (one SI is composed of one CCHI, and

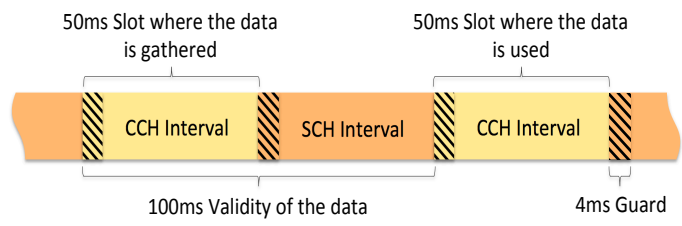

Figure 1: CCHI/SCHI alternation in IEEE802.11p showing the time interval when the data about the density of the network is gathered, and the time interval when it is used (100 to 150 ms later)

one SCHI) in order to broadcast their beacons. Considering that every vehicle has to broadcast its beacon at least once every CCHI, some works [3] argue that no more than 30 vehicles approximately can be in each other's reach (each vehicle can reach up to 30 vehicles as a maximum when broadcasting its beacon) in order to guarantee channel access to all vehicles.

The maximum speed in this context is limited to $50 \mathrm{~km} / \mathrm{h}$; the relative speed between two vehicles moving in opposite directions can reach up to $100 \mathrm{~km} / \mathrm{h}$ (approximately $28 \mathrm{~m} / \mathrm{s}$ ). This relatively high speed can emphasise the high dynamicity in such a network as one vehicle can observe a high variation in vehicles density around itself in as little as one SI. The small number of vehicles allowed to broadcast in each other's range (30 vehicles) adds up to this last fact to require an aggressive adaptation mechanism based on real time network data.

We also assume that each vehicle is equipped with a navigation system that enables positioning and time synchronization as well as with IEEE 802.11p [4] communication technology and computation capabilities. Each vehicle broadcasts 250 Bytes beacons periodically, which contain its id, its current position, its speed, the intended destination, and the number of vehicles observed ahead of it and behind it. The addition of these last two pieces of information in the beacon is possible since the standard specified in [14] defines the structure of the beacon with fields reserved for application specific uses.

\section{B. Short-term density prediction overview}

Usually in VANETs and from a single vehicle's perspective, each vehicle will broadcast a beacon message at least once every CCHI. Naturally, each vehicle will receive a number of beacon messages from its neighbours. The collection of these received beacons will indicate information about the neighbours like their ids (from which the percentage of new vehicles in the neighbourhood is deducted) their positions, speeds and destinations (from which is inferred the time each vehicle is sticking around before leaving the vicinity) and of course the number of beacons received (from which the density of vehicles around the receiver is deduced). Tx parameters are later adapted according to this observed density to control the channel congestion and reduce packet collisions.

The main purpose of using a prediction mechanism is allowing vehicles to regulate the flow of their beacons based on data reflecting the current situation of the network instead of basing it on deprecated data. According to the specifications 


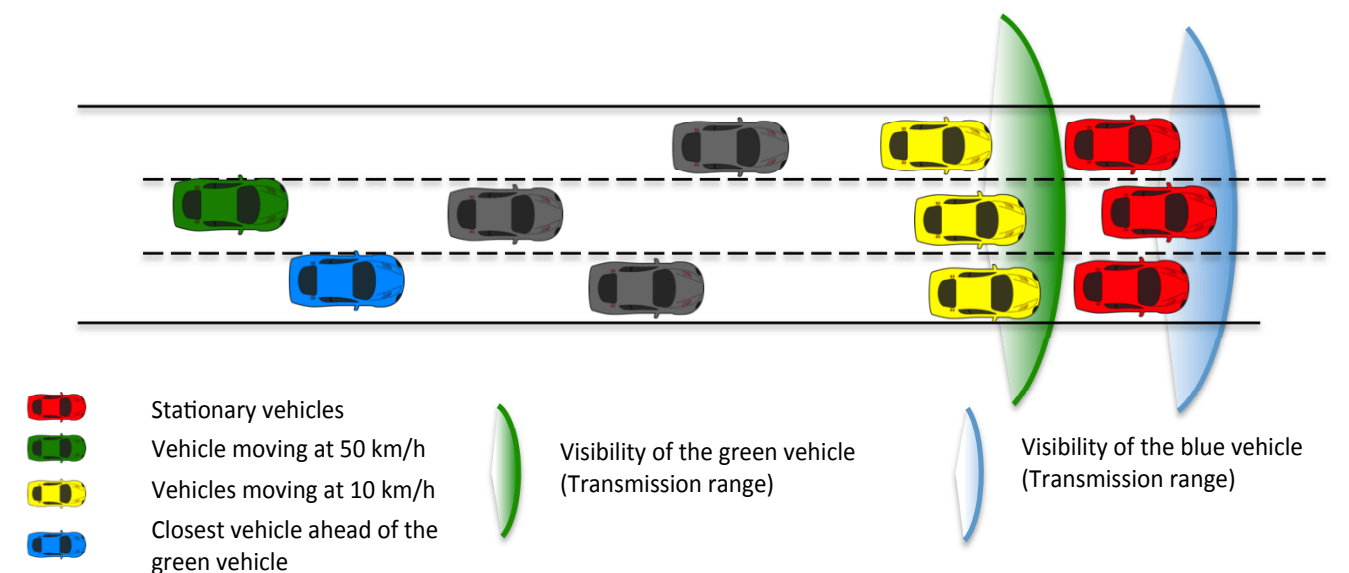

Figure 2: Prediction mechanism illustration with the green vehicle predicting the next density with the help of information received from the blue vehicle

introduced in [2], information contained in beacons is outdated after 100 milliseconds. Under the current assumptions of literature solutions and as shown in Figure 1, this delay can range between 100 and 150 milliseconds, which is not acceptable especially for safety applications.

Our solution is a vehicle-centric mechanism in which, each vehicle predicts the density of vehicles around itself within the next CCHI and use that information in the adequate time to adapt Tx parameters. The approach used in order to accomplish this prediction is pretty simple and straightforward.

In practice, the speed of vehicles ahead (or behind) is a good indicator for the short-term variation of the density around, as drivers usually tend to speed up where no obstacles are ahead and slow down whenever someone or something is blocking their way. Our density prediction scheme relies on this rule of thumb by setting each vehicle to observe in its transmission range the speeds of the furthest vehicles ahead and the furthest behind, and then comparing them to its own speed. If a speed difference is spotted, the receiver vehicle considers the density observed by the closest vehicle ahead of it. The concept is explained in Figure 2.

As it is shown in this figure, the green vehicle moves at 50 $\mathrm{km} / \mathrm{h}$ and has a visibility (transmission range) up to the yellow vehicles, which in turn move at $10 \mathrm{~km} / \mathrm{h}$. The blue vehicle is the closest vehicle moving ahead of the green one and has a visibility up to the red vehicles, which are stationary. The green vehicle has no imminent obstacles that might cause it to slow down while the yellow vehicles have already started slowing down because of the red ones. This indicates that if these same speeds are maintained (which is most likely in such a short time interval), the green vehicle will observe an increase in density in the short term since its transmission range will reach the red vehicles. The same applies if there are some vehicles behind the green one: if the speed of the furthest vehicles behind is higher, it is an indicator that some new vehicles might reach its transmission range from behind and therefore increase the density in its neighbourhood. Since the red vehicles are already visible to the blue one (they already are within its transmission reach), this latter can inform the green vehicle of the number of new vehicles it will count within its reach in the next short while.

As stated earlier in this section (III-A), two additional pieces of information are added to each beacon transmitted by each vehicle. The first one is the density of vehicles ahead (the number of vehicles which are ahead of the current vehicle with regard to its direction) and the second one is the density of vehicles behind.

When a vehicle receives beacons from the furthest vehicles ahead, it retrieves their speeds. If these speeds are lower than the receiver's speed it retrieves the density ahead (the first additional field in the beacon) from the closest vehicle ahead of it and includes it as its own predicted density ahead. The same is done with the beacons received from the furthest vehicles behind; if their speeds are higher than that of the receiver, this latter retrieves the density behind (the second additional field in the beacon) from the closest vehicle behind and counts it as its own predicted density behind. The sum of these two predicted densities (ahead and behind) is used to regulate Tx parameters during the next CCHI.

\section{Short-term density prediction algorithm}

In this subsection, we will explain the operation mode of the short-term density prediction algorithm.

Algorithm 1 shows how our short-term density prediction is performed. This algorithm is executed at the end of every $\mathrm{CCHI}$ and is node-centric i.e. it is carried out in each individual vehicle and based on the data it has observed and received.

First, each vehicle will compute the density of vehicles ahead and behind itself as observed from the beacons received from all the neighbours. The calculation method of these two densities is explained later in this subsection. Then, these two densities are appended to the beacon and the broadcast of this latter is scheduled for the next CCHI (approximately 50 to 100 milliseconds later). Next, from the beacons received from all neighbours, each vehicle will check if it has a close enough neighbour ahead of it (and/or behind it), which can inform it about the state of vehicle density in the next time window. If yes, this current vehicle will check if the conditions 
to include the density of the closest vehicle ahead/behind are met, namely a speed difference between the current vehicle and the furthest vehicles in the transmission range, or a change in the observed density compared to the last time window. Now that all conditions are met, this current vehicle will consider the density ahead of the closest vehicle ahead of it and the density behind of the closest vehicle behind it as its own predicted density for the next time window. The sum of these two densities will constitute the basis for the beacons' Tx parameters adaptation.

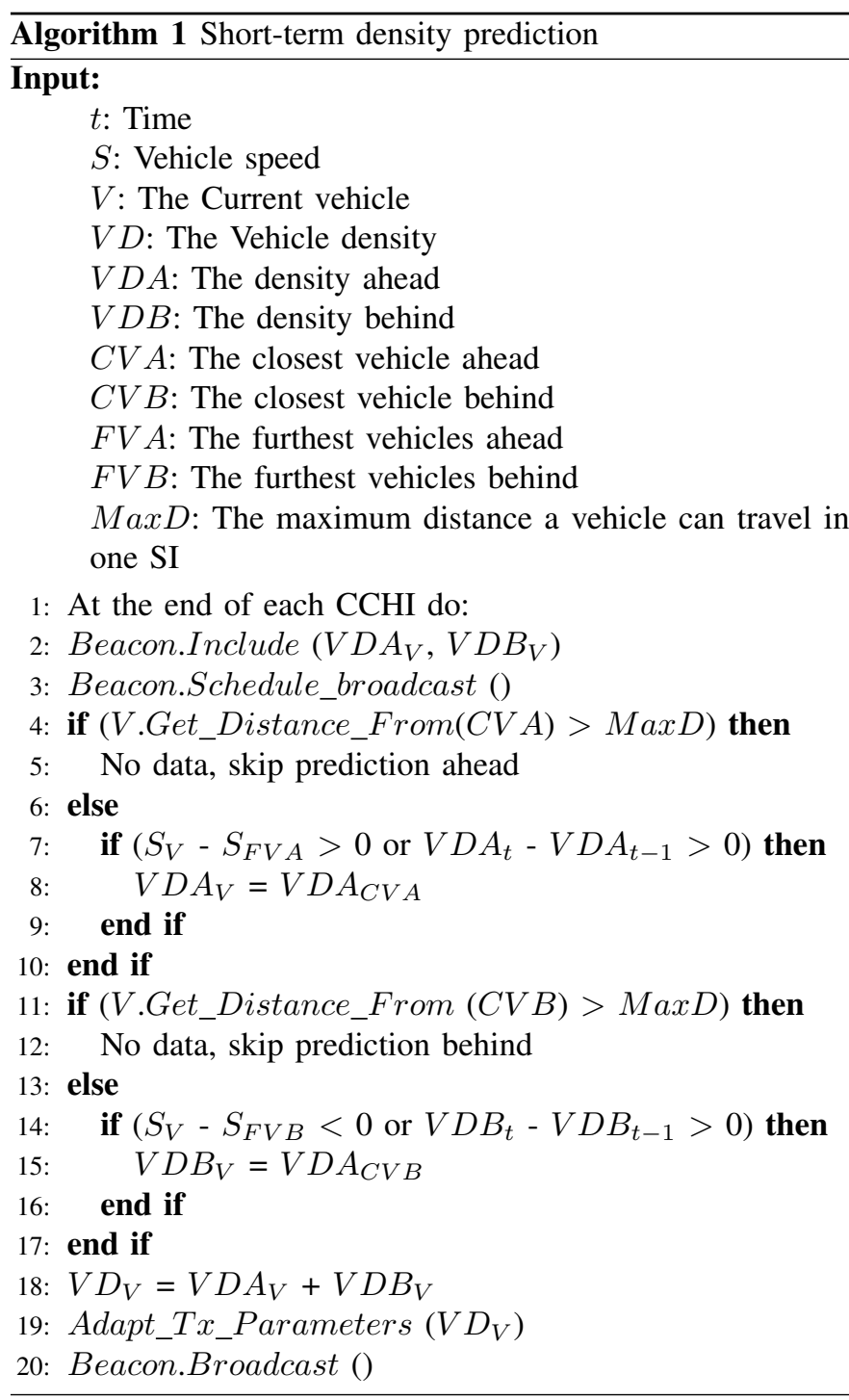

To perform this adaptation, this predicted density is then translated into an expected channel busy ratio. The rationale for this translation is simple: considering that each vehicle is expected to broadcast one or more beacons every CCHI, an increase or decrease in the density of vehicles competing for an access to the channel would impact the channel busy ratio in a direct way and a simple rule of three would give us the expected channel busy ratio. If for example an increase of $5 \%$ is expected in the density of vehicles within the next time window, an equivalent increase is expected in terms of channel busy ratio and vice versa. We refer the reader to [3] for more details about the adaptation algorithm used.

In order to compute the density ahead and/or behind, each vehicle will need to check every beacon it receives and determine whether it is from a vehicle ahead of it with regard to its movement and direction, or from a vehicle that is behind it. To this end, vehicles need to perform a certain number of calculations, which can be demonstrated in Figure 3.

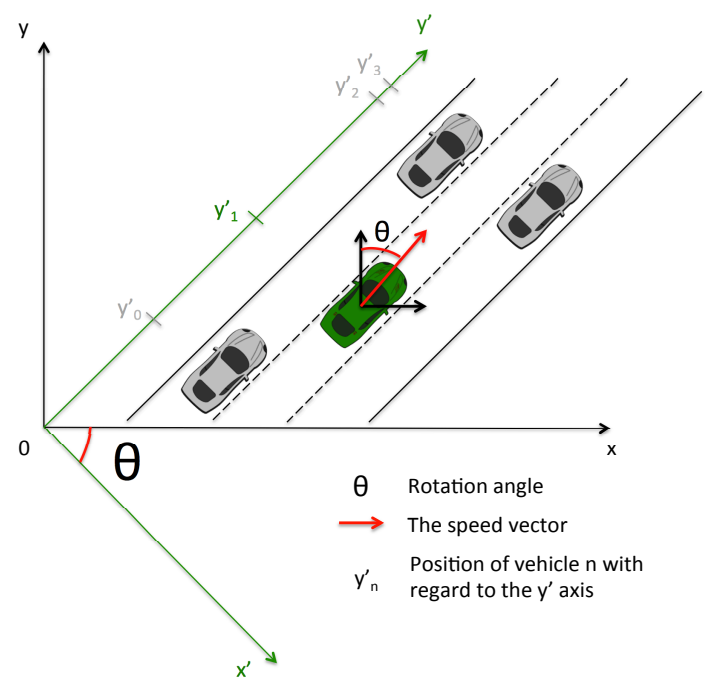

Figure 3: 2D axes rotation to define the set of vehicles ahead and the set of vehicles behind of the green vehicle with regard to its direction

As it can be seen in Figure 3, the angle of the road, and thus that of vehicles, might not always be parallel to one of the coordinates axis. After translating the latitude longitude coordinates to Cartesian coordinates, we perform a 2D rotation of the $(x, y)$ axes with an angle $\theta$ that represents the shifting needed to make the y-axis parallel to the speed vector of the vehicle. The angle $\theta$ is calculated as follows:

$$
\theta= \begin{cases}\tan ^{-1}\left(\frac{\Delta y}{\Delta x}\right)-\frac{\pi}{2} & \text { if } x>0 \\ \tan ^{-1}\left(\frac{\Delta y}{\Delta x}\right)+\frac{\pi}{2} & \text { otherwise }\end{cases}
$$

where $\Delta x$ and $\Delta y$ are the $x$ and $y$ components of the speed vector.

Next, we compute the relative coordinates to the new $\left(x^{\prime}\right.$, $y^{\prime}$ ) axes pair using the following formulas:

$$
\begin{aligned}
& x^{\prime}=x \cos (\theta)-y \sin (\theta) \\
& y^{\prime}=y \cos (\theta)+x \sin (\theta)
\end{aligned}
$$

Once the new $\left(x^{\prime}, y^{\prime}\right)$ pair is calculated, we simply compare vehicles' $y^{\prime}$ coordinates. $y_{A}^{\prime}$ greater than $y_{B}^{\prime}$ means that vehicle $A$ is ahead of vehicle $B$ and vice versa.

\section{Performance EVAluation}

In this section, we present the results after comparing our scheme with four other schemes namely SuRPA (Successive Rate and Power adaptation [3]) without the density prediction, and three variants of DCC (Decentralised Congestion Control), 
which are ETSI-TRC (Transmit Rate Control), ETSI-TPC (Transmit Power Control) and ETSI-DCC (TRC and TPC combined). We refer the reader to [1] for more information about DCC.

\section{A. Simulation setting}

To make sure our tests are the closest possible to reality, we conducted our simulations in a realistic VANET environment. The mobility of vehicles is generated using SUMO ${ }^{1}$ and the communication between them are ensured using NS-3 ${ }^{2}$. This latter offers the possibility to use the IEEE802.11p MAC layer with $10 \mathrm{MHz}$ channels. Both simulators run simultaneously using HINTS platform [15] allowing real time information exchange and increased degree of realness in the simulation. Furthermore, a realistic radio propagation model that takes into account the effects of shadowing caused by buildings, namely the Corner model [16] was chosen to mimic the transmission of messages in urban areas. The full list of parameters used in our simulation can be found in Table I.

Table I: Simulation parameters

\begin{tabular}{ccc}
\hline & Frequency band & $5.9 \mathrm{GHz}$ \\
BHY & Bandwidth & $10 \mathrm{MHz}$ \\
& Transmission range & $260 \mathrm{~m}$ \\
& Max/Min Tx Power & $19.03 / 5 \mathrm{dBm}$ \\
& Receiver sensitivity & $-45 \mathrm{dBm}$ \\
& Noise & $-105 \mathrm{dBm}$ \\
\hline \multirow{3}{*}{ LLC } & Bit rate & $6 \mathrm{Mbits} / \mathrm{s}$ \\
& CW & {$[3,7]$} \\
& AIFSN & 2 \\
\hline \multirow{3}{*}{ ETSI } & Relaxed/Active/restrictive & $19.03 / 15 /-10 \mathrm{dBm}$ \\
& Relaxed/Active/restrictive & $25 \mathrm{~Hz} / 2 \mathrm{~Hz} / 1 \mathrm{~Hz}$ \\
& Max /Min Channel load & $0.4 / 0.15$ \\
\hline \multirow{5}{*}{ Prediction } & Data message size & $256 \mathrm{Bytes}$ \\
& Max/Min beacons freq & $50 \mathrm{~Hz} / 10 \mathrm{~Hz}$ \\
& Acceptable collision rate & 0.05 \\
& Desired channel load & 0.3 \\
& MaxD & $2 \mathrm{~m}$ \\
\hline
\end{tabular}

Building on this simulation configuration, we implemented our prediction scheme on top of SuRPA, as well as the four other schemes cited above, namely SuRPA without the density prediction, ETSI-TRC, ETSI-TPC and ETSI-DCC, to compare against. We picked a road map composed of $1.2 \mathrm{~km}$ roads crossing in the middle. Each road has 3 lanes in each direction and is populated with different vehicle types (cars, trucks, busses, etc.). We performed several runs of 1800 seconds to obtain more accurate results.

\section{B. Short-term prediction evaluation}

The first metric we chose in order to showcase the efficiency of our prediction scheme is the deviation (Figure 4) of the observed/predicted density from the real density around each individual vehicle. Each vehicle in the simulation observes (and predicts in our scheme) the density of vehicles around itself. This density is compared with the effective density around the given vehicle, to show the amount of deflection from the effective density, as a deviation of $0 \%$ is the best case

${ }^{1}$ http://www.sumo-sim.org/

${ }^{2}$ http://www.nsnam.org scenario and the higher the deviation the worse are the results. This deviation is calculated using the following formula:

$$
\text { Deviation }=100-100 \times\left(\frac{\sum \text { observed_density }}{\sum \text { real_density }}\right)
$$

Where the observed_density is the density observed (predicted in our scheme) by each individual vehicle, and the real_density is the effective density retrieved from our simulation. The results shown in figure 4 are obtained using the mean value of vehicles' densities among all vehicles, and retrieved for different vehicular densities in the simulation (the total number of vehicles in the simulation).

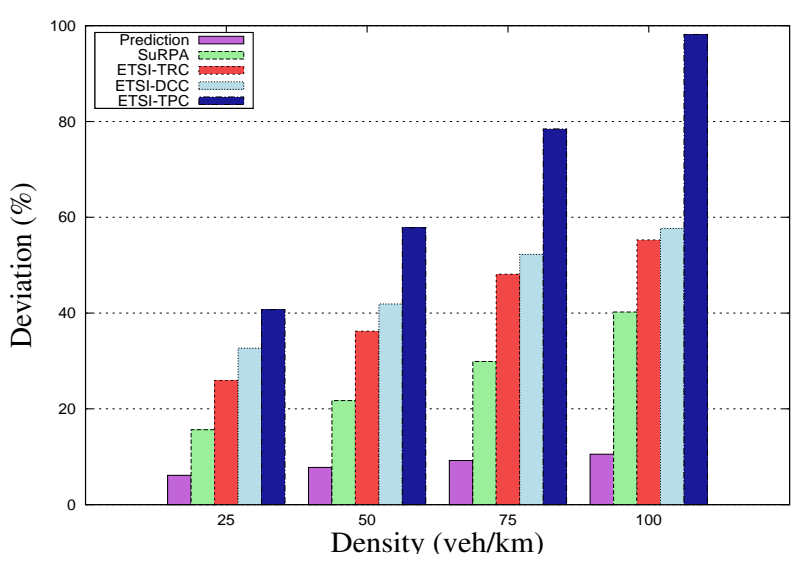

Figure 4: The percentage of deviation from the effective (real) case under different vehicle densities: Prediction vs. SuRPA and ETSI schemes

This first set of results show a clear advantage for our prediction scheme compared to the other schemes with a deviation of $6 \%$ at a density of 25 vehicles $/ \mathrm{km}$ against $15 \%$ for SuRPA and up to $40 \%$ for ETSI-TPC. The same pattern is observed as the density of vehicles increases in the simulation, the gap gets bigger as we recorded $10 \%$ deviation for our scheme at a density of 100 vehicles $/ \mathrm{km}$ against $40 \%$ for SuRPA and up to $98 \%$ for ETSI-TPC.

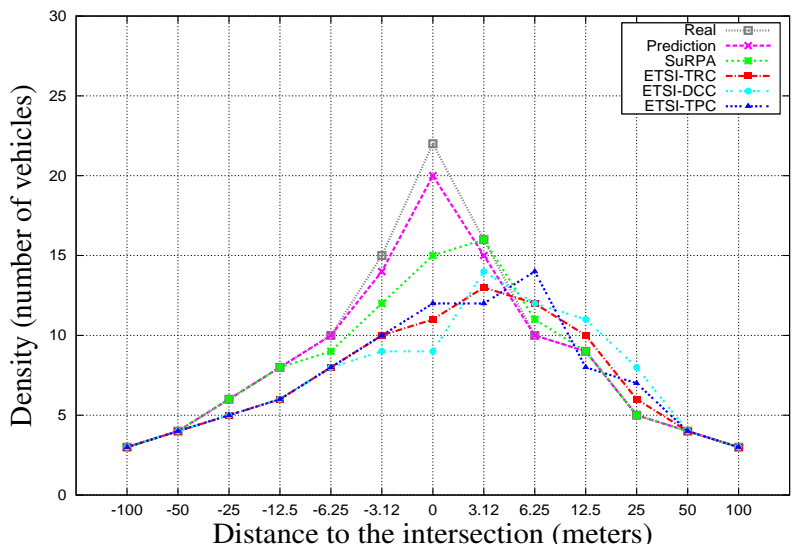

Figure 5: Impact of the distance to the intersection on the observed density: Prediction vs. SuRPA and ETSI schemes 


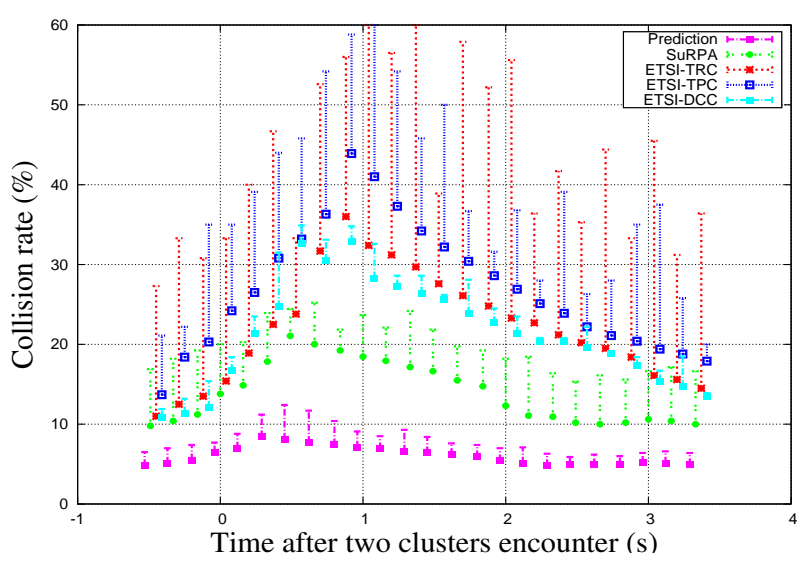

(a) Collision Rate vs. density

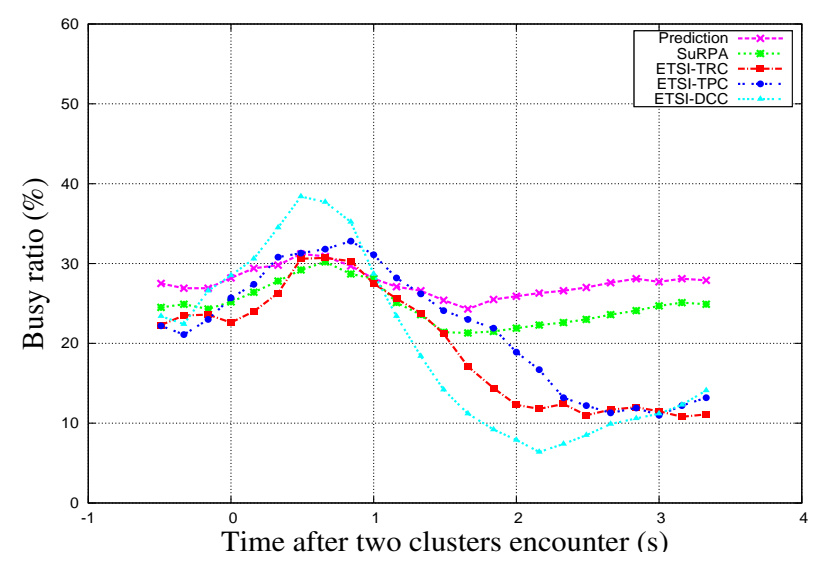

(b) Busy ratio vs. density

Figure 6: Impact of vehicles density on the achieved collision rate and channel busy ratio: Prediction vs. SuRPA and ETSI schemes

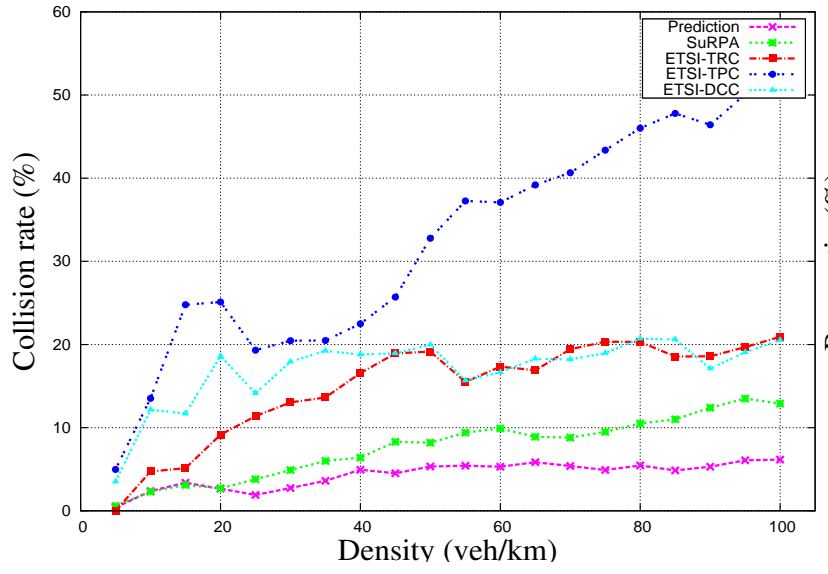

(a) Collision Rate vs. time

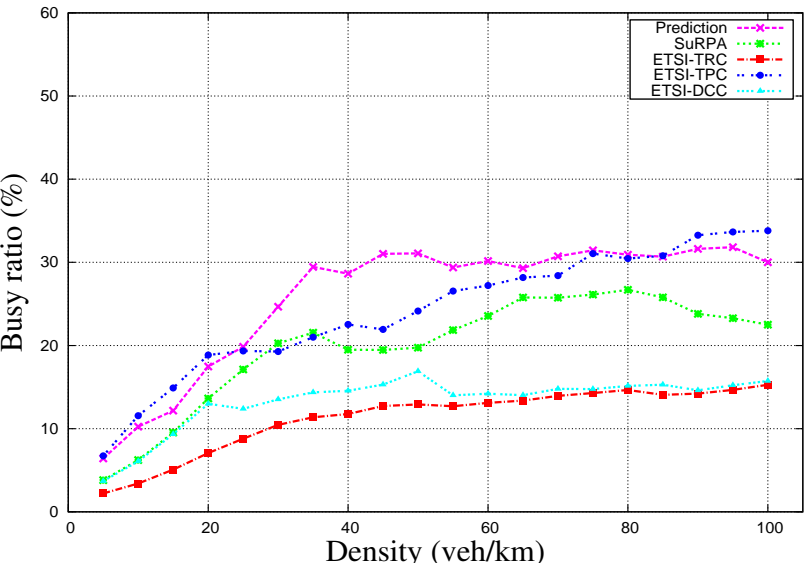

(b) Busy ratio vs. time

Figure 7: Tx parameters variation after two clusters of vehicles meet: Prediction vs. SuRPA ETSI schemes

Figure 5 shows the benefit we obtained using our scheme in terms of observed (predicted in our case) density from a single vehicle's perspective. We retrieved this observed (predicted) density from vehicles moving towards the intersection and crossing it. Here again, our density prediction scheme performs much better than the four other schemes, as it is the closest to the real density, especially in the area closer to the intersection where the variation of the density is faster and more important.

This metric is also, like the first one, meant to show the accuracy of our short-term vehicular density prediction scheme in both a macroscopic point of view in the first as well as a microscopic one in the second. The results show a substantial improvement in terms of preciseness of the solution with regard to the effective case compared to the other schemes. It is worth to call to mind the importance of an accurate vision of the network. An outdated or biased density observed by vehicles would result in an erroneous adaptation of the transmission parameters, which will in turn cause poor network performance like high packet losses and misuse or underutilisation of the available bandwidth. This will lead us to our next set of results.
Figure 6 reveals the impact of vehicle density on the collision rate and the busy ratio in our scheme as well as in all other four schemes. Figure 6(a) shows how our short-term prediction scheme keeps the collision rate below the threshold of $10 \%$ in all densities while SuRPA fluctuates around this threshold reaching up to $13 \%$ in higher densities, and the ETSI schemes reach limits that are not tolerated for VANETs and safety applications in particular. Similarly, Figure 6(b) shows the variation of the busy ratio with the increase of density. We can see that the prediction scheme allows a non-negligible improvement in terms of channel usage as it fluctuates around $30 \%$ surpassing SuRPA and the other ETSI schemes. We notice that ETSI-TPC achieves a slightly higher busy ratio with densities higher than 80 vehicles $/ \mathrm{km}$, but this can be discarded since this same scheme has a collision rate higher than $45 \%$ at this density level.

Finally, Figure 7 shows the variation of the collision rate and the busy ratio when two clusters of vehicles meet. In Figure $7(a)$, we observe a considerable increase in the collision rate of all schemes, then slowly returning to the normal rates after a few seconds. While this increase remains very small for the 
short-term prediction scheme, it fluctuates to high values: $20 \%$ for SuRPA and up to $40 \%$ in some of the ETSI schemes. Also, our scheme gets back to its normal rate after approximately 1 second while the four other schemes take a few seconds longer to achieve that. Figure 7(b) on the other hand shows the variation of the busy ratio and demonstrates the stability of our new scheme compared to the other schemes as it shows the least variation among all five, while remaining close to the $30 \%$ threshold.

The two previous set of results (Figures 6 and 7) highlight the benefit we obtained after applying our short-term density prediction scheme in terms of network performance. It is needless to say how critical it is to achieve good overall network performance, due to the critical nature of applications that are meant to ensure the safety of drivers on the road. However, we would like to stress on the importance of such good performances in specific times and a microscopic perspective. In fact, an aggressive reaction to the changes in the network is crucial in VANETs, but this will never be complete without a proper density prediction mechanism to ensure more accurate data about the network state. And this is what we have showcased in this section first, by demonstrating the preciseness of our short-term vehicular density prediction; then by displaying the repercussion of this latter on the performance of the network.

\section{CONCLUSION AND Future Work}

In this paper, we presented a short-term vehicular density prediction scheme that aims at providing more accurate and up-to-date information about the network state. This prediction then helps to perform an improved adaptation to the transmission parameters in order to achieve better overall network performance. Simulations were conducted using a realistic simulation environment in terms of both channel conditions and vehicle mobility. The obtained results have proven the efficiency and the effectiveness of our scheme that enabled a significant enhancement in terms of channel busy ratio and successful packet delivery. This work helps the vehicular networks research community to gain deeper understanding as to why a density prediction is needed in such a network, and will open new research prospects by bringing up new challenges to VANETs research field.

\section{ACKNOWLEDGMENT}

This work was supported, in part, by Science Foundation Ireland Grant 10/CE/I1855 and by Science Foundation Ireland grant 13/RC/2094 to Lero, the Irish Software Engineering Research Centre (www.lero.ie). This work was also supported by the Earth and Natural Sciences Doctoral Studies Programme, which is funded by the Higher Education Authority under the Programme for Research in Third- Level Institutions
(PRTLI) Cycle 5 and co-funded under the European Regional Development Fund (ERDF).

\section{REFERENCES}

[1] ETSI TS 102687 V1.1.1, "Intelligent Transport Systems (ITS); Decentralized Congestion Control Mechanisms for Intelligent Transport Systems operating in the $5 \mathrm{GHz}$ range; Access layer part," July 2011

[2] CAMP Vehicle Safety Communications Consortium. (2005). Vehicle safety communications project task 3 final report: Identify intelligent vehicle safety applications enabled by DSRC, Nat. Highway Traffic Safety Admin., U.S. Dept. Transp. (USDOT), Washington, DC, Tech. Rep. DOT HS 809859. [Online]. Available: http://www.its.dot.gov/research_docs/ pdf/59vehicle- safety.pdf

[3] S. Zemouri, S. Djahel, and J. Murphy. "Smart adaptation of beacons transmission rate and power for enhanced vehicular awareness in VANETs." in IEEE 17th International Conference on. Intelligent Transportation Systems (ITSC), Oct. 2014

[4] "Wireless LAN Medium Access Control (MAC) and Physical Layer (PHY) Specifications Amendment 6: Wireless Access in Vehicular Environments," in IEEE, Standard 802.11p-2010, 2010.

[5] C. Sommer, O. K. Tonguz, and F. Dressler, "Traffic Information Systems: Efficient Message Dissemination via Adaptive Beaconing," in IEEE Communications Magazine, vol. 49, no. 5, pp. 173-179, 2011.

[6] C. Sommer, S. Joerer, M. Segata , O. Tonguz, R. L. Cigno, and F. Dressler, "How shadowing hurts vehicular communications and how dynamic beaconing can help", in INFOCOM, April 2013.

[7] M. Torrent-Moreno, J. Mittag, P. Santi, andH. Hartenstein, "Vehicle-tovehicle communication: fair transmit power control for safety-critical information", in Vehicular Technology, IEEE Transactions on, 58(7), (2009) 3684-3703.

[8] Kloiber, B., Härri, J., Strang, T., Hrizi, F., Bonnet, C., Rico-Garcia, C., ... \& Blokpoel, R. "Dice the TX power-Improving Awareness Quality in VANETs by random transmit power selection," in VNC, 2012 (pp. 56-63).

[9] Sander Frigau, M. "Cross-layer transmit power and beacon rate adaptation for VANETs," in Proceedings of the third ACM international symposium on Design and analysis of intelligent vehicular networks and applications, Nov. 2013, (pp. 129-136). ACM.

[10] Baldessari, R., Scanferla, D., Le, L., Zhang, W., \& Festag, A. "Joining forces for vanets: A combined transmit power and rate control algorithm," in 6th International Workshop on Intelligent Transportation (WIT), March 2010.

[11] Huang, C. L., Fallah, Y. P., Sengupta, R., \& Krishnan, H. "Adaptive intervehicle communication control for cooperative safety systems," in Network, IEEE, 2010, 24(1), 6-13.

[12] Autolitano, A., Campolo, C., Molinaro, A., Scopigno, R. M., \& Vesco, A. "An insight into Decentralized Congestion Control techniques for VANETs from ETSI TS 102687 V1. 1.1, " in Wireless Days (WD), Nov. 2013 IFIP(pp. 1-6). IEEE.

[13] Eckhoff, D., Sofra, N., \& German, R. "A performance study of cooperative awareness in ETSI ITS G5 and IEEE WAVE," in Wireless On-demand Network Systems and Services (WONS), 2013 10th Annual Conference on, March 2013, (pp. 196-200). IEEE.

[14] "Standard Specification for Telecommunications and Information Exchange Between Roadside and Vehicle Systems $5 \mathrm{GHz}$ Band Dedicated Short Range Communications (DSRCU) Medium Access Control (MAC) and Physical Layer (PHY) Specifications", ASTM E2213-03, 2010.

[15] S. Zemouri, S. Mehar \& S.M. Senouci, "HINTS: A novel approach for realistic simulations of vehicular communications," in Global Information Infrastructure and Networking Symposium (GIIS), Dec. 2012

[16] A. Mukunthan, C. Cooper, F. Safaei, D. Franklin \& M. Abolhasan, "Studying the Impact of the CORNER Propagation Model on VANET Routing in Urban Environments," in IEEE Vehicular Technology Conference (VTC Fall), Sep. 2012 